\title{
Comparison of five percutaneous pinning methods for unstable extra-articular distal radius fractures: A mechanical study using sawbones
}

\author{
S. Kıvanç Muratlı, MD'1D, Bora Uzun, $\mathrm{PhD}^{2}$ (D), Salih Çelik, $\mathrm{MSc}, \mathrm{BSc}^{3}$ (I) \\ 'Department of Orthopedics and Traumatology, Çanakkale Onsekiz Mart University, School of Medicine, Çanakkale, Turkey \\ ${ }^{2}$ Department of Biomechanics, Dokuz Eylül University, School of Medicine, Izmir, Turkey \\ 3University of Bonn, Zentrum für Zahn-, Mund,- und Kieferheilkunde, Bonn, Germany
}

Displaced extra-articular distal radius fractures are generally treated by closed reduction and plaster immobilization methods. However, in patients with unstable fractures, reduction loss is frequently observed and surgical methods may be necessary to prevent malunion. Distal radius fractures with dorsal comminution are unstable fractures common in this area. Surgical treatment of these fractures include fixation with percutaneous Kirschner wires (K-wire), external fixation, internal fixation with various types of implants, and combinations of these methods.

Closed reduction and percutaneous K-wire fixation is a relatively less invasive and lower cost method for treatment of these types of fractures and as such, is an attractive choice in certain

Received: April 28, 2020

Accepted: September 21, 2020

Published online: January 06, 2021

Correspondence: S. Kıvanç Muratı, MD. Çanakkale Onsekiz Mart Üniversitesi Tıp Fakültesi Ortopedi ve Travmatoloji Anabilim Dalı, 17100 çanakkale, Türkiye.

E-mail: skmuratli@hotmail.com

Doi: $10.5606 /$ ehc. 2021.75817

Citation: Muratlı SK, Uzun B, Celik S. Comparison of five percutaneous pinning methods for unstable extra-articular distal radius fractures: A mechanical study using sawbones. Jt Dis Relat Surg 2021;32(1):51-58.

(O2021 All right reserved by the Turkish Joint Diseases Foundation

This is an open access article under the terms of the Creative Commons Attribution-NonCommercial License, which permits use, distribution and reproduction in any medium, provided the original work is properly cited and is not used for commercial purposes (http://creativecommons.org/licenses/by-nc/4.0/).

\section{ABSTRACT}

Objectives: This study aims to mechanically compare five different extra-focal bi-cortical pin configurations (using two and three pins) employed for fixation of a simulated unstable extra-articular distal radius fracture with dorsal comminution using a sawbone model.

Materials and methods: This in vitro mechanical study was conducted between June 2019 and July 2019. A standard fracture model (Arbeitsgemeinschaft für Osteosynthesefragen [AO] type 23-A3.3) was created using a fourth generation composite artificial radius bone. Five groups with two- and three-pin configurations were tested under axial, volar, and dorsal loading with a universal test device. Mean stiffness values were compared statistically.

Results: Comparison of stiffness values from axial and volar loading tests between groups in paired comparison showed no statistically significant difference $(p=0.194$ and $p=0.086$, respectively). Dorsal loading tests showed statistically significant difference between the groups in pairwise comparison ( $\mathrm{p}=0.002)$. Three-pin groups (Groups 3, 4, and 5) had higher stiffness values compared to two-pin groups (Groups 1 and 2) in dorsal loading tests $(\mathrm{p}=0.001)$. Three-pin configuration test groups with two divergent or convergent pins from the radial styloid performed better compared to both two-pin groups $(\mathrm{p}=0.01, \mathrm{p}=0.002)$ in dorsal loading tests.

Conclusion: Our data demonstrated that the three-pin configuration with two divergent or convergent Kirschner wires from the styloid and a third wire from the dorsal/ulnar cortex had higher stiffness values compared to two-pin configurations in dorsal loading tests. When indicated, we suggest the use of a three-pin construct. Particularly in cases with a risk of volar angulation, we recommend a three-pin configuration with two divergent or convergent bi-cortical Kirschner wires.

Keywords: Biomechanics, distal radius, fracture, percutaneous pinning, sawbones, three-pin, two-pin.

conditions. ${ }^{[1,2]}$ Several methods are recognized for fixation of these fractures, $(i)$ using extra-focal wires fixed to both distal and proximal fragment cortices from the radial styloid (Willenegger's method); ${ }^{[3]}$ 
(ii) using intra-focal wires introduced from the fracture line to the cortex of proximal fragments (Kapandji's intrafocal method); ${ }^{[4]}$ (iii) from the cortex of distal fragments toward the proximal with wires through the intramedullary canal (Py's isoelastic method) $i_{i}^{[5,6]}$ (iv) or a combination of these methods. There are many clinical studies concerning the percutaneous fixation of extra-articular distal radius fractures in the literature..$^{[1,2,7-9]}$ However, there are only a limited number of controlled experimental biomechanical studies focusing on this topic. ${ }^{[7,8,10]}$ Furthermore, biomechanical studies focusing on the pin numbers and/or pin configurations are quite sparse. Within the past several decades, the rate of surgical management of distal radius fractures particularly with volar locking plates seems to be increasing. Compared to percutaneous pinning, volar locking plates provide better early functional results, while in the long-term, follow-ups of both volar locking plates and closed reduction and percutaneous pinning demonstrate excellent functional results..$^{[11,12]}$ The American Academy of Orthopaedic Surgeons clinical guideline for the treatment of distal radius fractures has 29 recommendations rating between moderate and inconclusive strength levels, and none of them was able to have a strong level of strength. The fourth recommendation about choosing a specific operative method and $16^{\text {th }}$ recommendation about whether two or three K-wires should be used for distal radius fracture fixation have both inconclusive results. ${ }^{[13]}$ While reminding the effectiveness of percutaneous pinning, we planned to investigate if there is a difference between two- and three-pin configurations and demonstrate in which configurations the extra-focal percutaneous pinning is most stable. The extra-focal bi-cortical pin configurations were chosen as being commonly used and are generally accepted in the literature to provide stable fixation.

We hypothesized that the stability of the fixation of the fracture site would be better in three-pin configurations when compared to two-pin configurations. We also hypothesized that, particularly under axial loading, the stability would be the higher in pin configurations having two convergent or divergent pins on the radial styloid. We thought that the unparallel configuration of the pins would have better resistance particularly against axial loads to minimize radial shortening compared to parallel pins. Therefore, in this study, we aimed to mechanically compare five different extra-focal bi-cortical pin configurations (using two and three pins) employed for fixation of a simulated unstable extra-articular distal radius fracture with dorsal comminution using a sawbone model.

\section{MATERIALS AND METHODS}

This in vitro mechanical study was conducted at Dokuz Eylül University School of Medicine, Biomechanics Laboratory between June 2019 and July 2019. Five pin configuration groups, two groups with two pins and three groups with three pins, were chosen to investigate the stability of percutaneous fixation of simulated extra-articular distal radius fractures using a total of 35 (seven in each group) sawbones. Group 1: Only two parallel Kirschner wires introduced from the radial styloid diagonally crossing the fracture line and fixed to the facing cortex of the proximal fragment (Figure 1). Group 2: One K-wire introduced from the radial
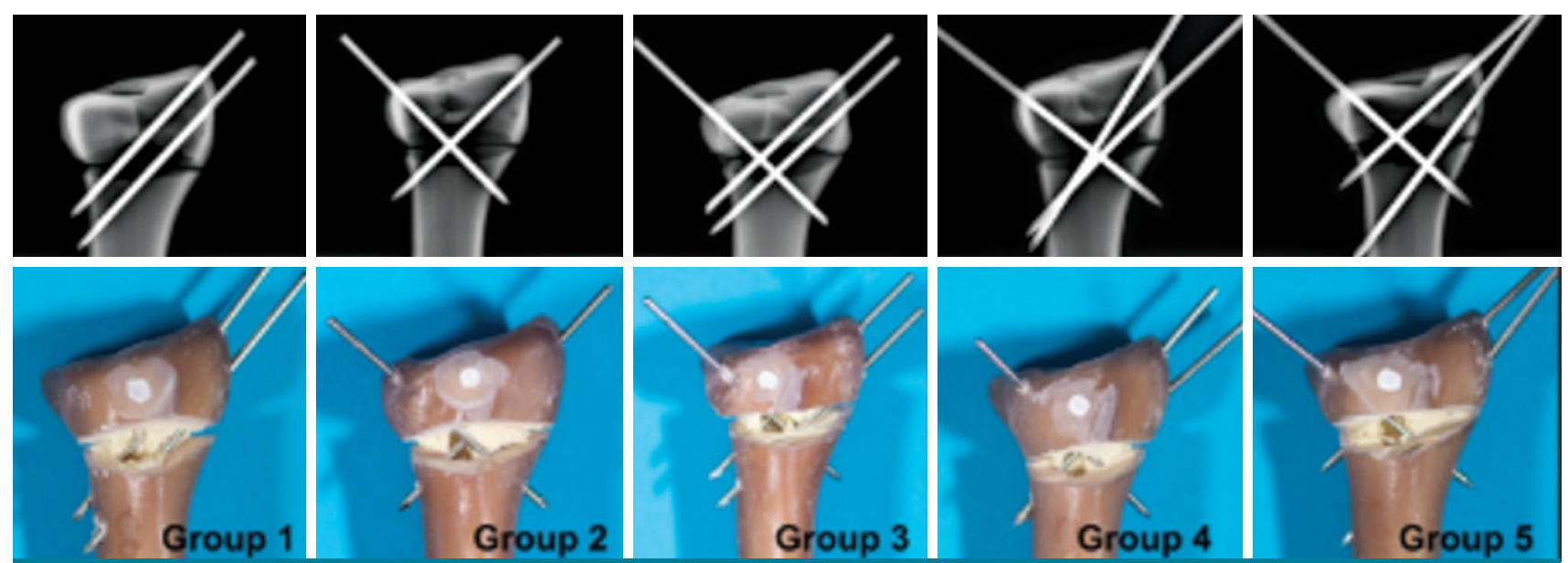

FIGURE 1. Anteroposterior X-ray views and photographs of pin configurations of each study group. 


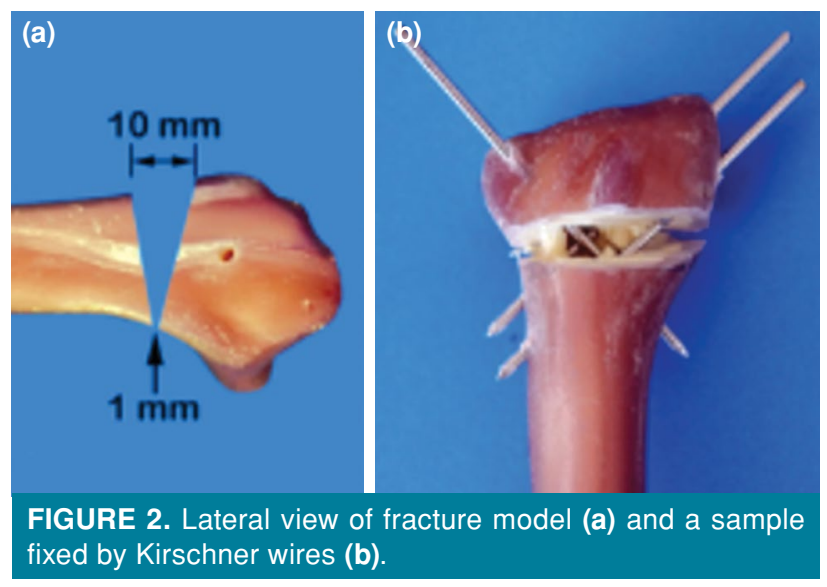

styloid diagonally crossing the fracture line and fixed to the facing cortex of the proximal fragment. The second K-wire introduced from the ulnar portion of the dorsal metaphyseal cortex diagonally crossing the fracture line and previously introduced K-wire and fixed to the cortex of the proximal fragment (Figure 1). Group 3: Two parallel K-wires introduced from the radial styloid diagonally crossing the fracture line and fixed to the facing cortex of the proximal fragment. The third K-wire introduced from the ulnar portion of the dorsal metaphyseal cortex diagonally crossing the fracture line and previously introduced K-wires and fixed to the cortex of the proximal fragment (Figure 1). Group 4: Two convergent K-wires introduced from the radial styloid diagonally crossing the fracture line and fixed to the facing cortex of the proximal fragment. The third K-wire introduced from the ulnar portion of the dorsal metaphyseal cortex diagonally crossing the fracture line and previously introduced K-wires and fixed to the cortex of the proximal fragment (Figure 1). Group 5: Two divergent K-wires introduced from the radial styloid diagonally crossing the fracture line and fixed to the facing cortex of the proximal fragment. The third K-wire introduced from the ulnar portion of the dorsal metaphyseal cortex diagonally crossing the fracture line and previously introduced K-wires and fixed to the cortex of the proximal fragment (Figure 1). Seven fourth generation composite radii (Item \#3407, Sawbones ${ }^{\circledR}$, Sawbones Europe AB Inc., Limhamn, Sweden) were used in each group. The radial styloid entry points were located on the dorsal prominence at the interval between the first and second extensor compartments as used in clinical practice. In some clinical situations, volar prominence may be preferred. The dorsal/ulnar located K-wire is usually introduced through the interval between the fourth and fifth extensor compartments and enters the dorsal cortex near the periphery of triangular fibrocartilaginous complex attachment. ${ }^{[14,15]}$

To simulate an extra-articular distal radius fracture with dorsal comminution (Arbeitsgemeinschaft für Osteosynthesefragen [AO] type 23-A3.3), a wedge osteotomy was performed $20 \mathrm{~mm}$ proximal from the joint surface. On the lateral view, the wedge had a $10 \mathrm{~mm}$ dorsal base and $1 \mathrm{~mm}$ volar surface peak-point gap. Osteotomy cuts and K-wire entry and exit points were standardized using guides and a digital
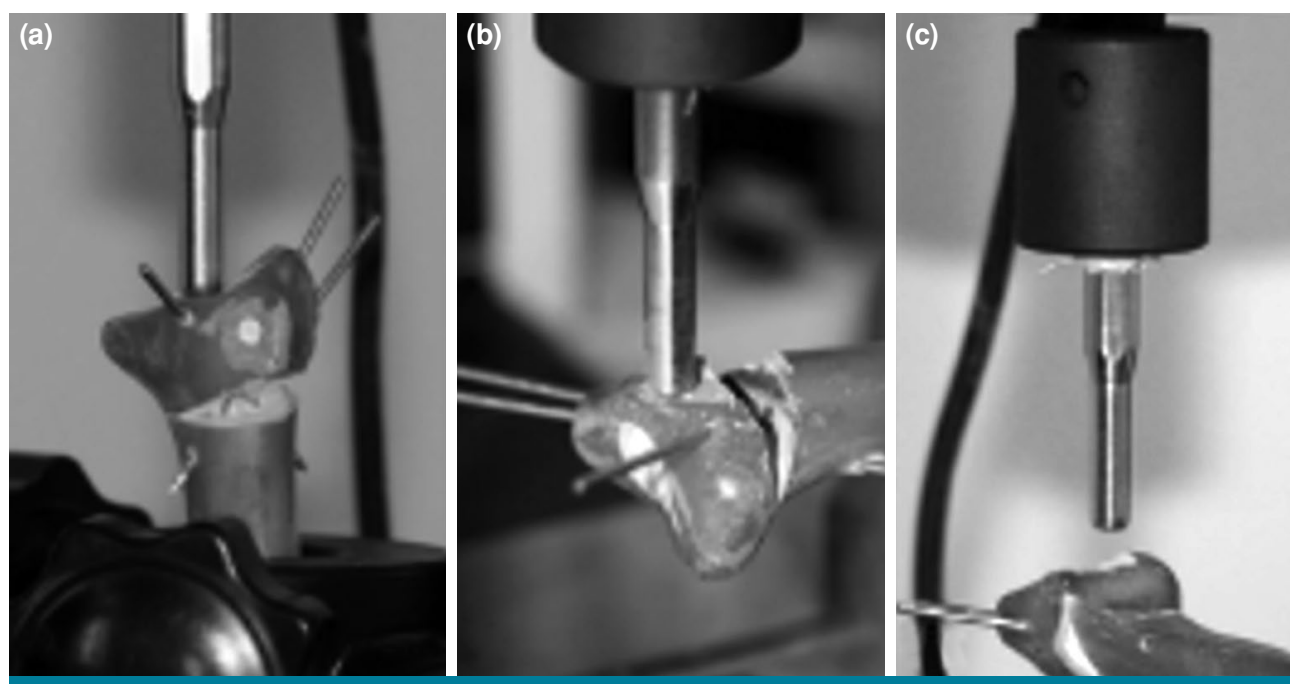

FIGURE 3. Loading points of axial loading tests (a), dorsal loading (volar bending) tests (b), and volar loading (dorsal bending) tests (c). 
Axial loading
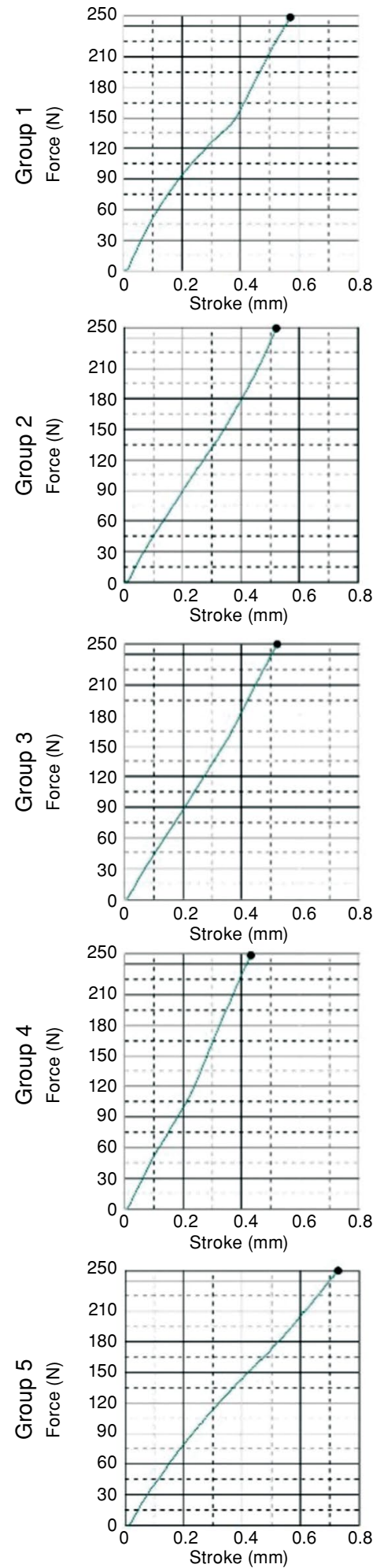
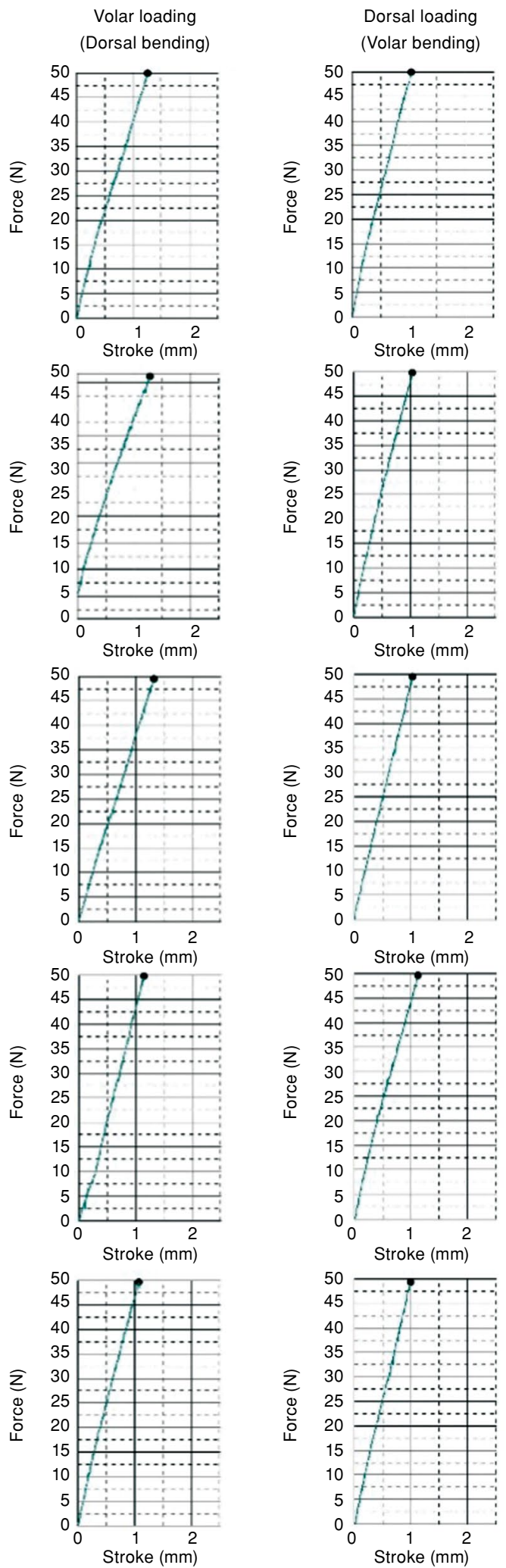

FIGURE 4. Samples of load-displacement curves of each study group. Minimal deviations in axial loading curves are due to vectoral alterations while closing of $1 \mathrm{~mm}$ volar fracture gap under compression. 
Vernier caliper (Figure 2). To prevent sliding of the device probe and inappropriate positioning, the loading points of the samples were processed to be congruent with the device probe using a power tool with an abrasive disc and guide points were marked precisely with a white marker. For fixation of the samples, $1.8 \mathrm{~mm}$ (0.070 inch) standard trocar-point smooth K-wires (Hipokrat Tibbi Malzemeler İmalat ve Pazarlama A.Ş., Izmir, Turkey. Grade X 2 CrNiMo AISI 316L/1.4441 in ASTM F138-03, ISO 5832-1 [last revision] standards. Test certificate: EN 10 204/3.1. Grain size in accordance with ASTM E 112: 10 and thinner, microscopic cleanliness in accordance with ISO 4967/ASTM E 45, method: A) were used.

The tests were completed using a universal test device (Shimadzu ${ }^{\circledR}$ Autograph AG-IS $5 \mathrm{kN}$, Load Cell: SLBN5KN, Shimadzu Co., Kyoto, Japan). Testing order of the samples were standardized as (i) axial loading, (ii) dorsal loading (volar bending), and (iii) volar loading (dorsal bending) for each sample. As non-load-to-failure loading modality was used and preliminary tests demonstrated no plastic deformation, all samples were subjected to axial loading $(250 \mathrm{~N})$, dorsal loading $(50 \mathrm{~N})$, and volar loading $(50 \mathrm{~N})$ as described in the study of Willis et al. ${ }^{[16]}$ Load rate for all samples in axial loading, dorsal loading, and volar loading was $5 \mathrm{~mm} / \mathrm{min}$ (at $50 \mathrm{~ms} / 200 \mathrm{~Hz}$ data rate).

Positioning and stabilization of the samples were performed by a custom-made pedestal (designed for the fixation of the composite radius material from both intramedullary and extramedullary sides) and clamps attached to the universal test device. Axial loading was applied to the samples at the intersection point of lunate and scaphoid fossae. Dorsal and volar loads were applied on the dorsal and volar cortices of the distal fragment at midline, $5 \mathrm{~mm}$ from the articular surface on the dorsal side, and $2 \mathrm{~mm}$ from the articular surface on the volar side (Figure 3).

\section{Statistical analysis}

Statistical analysis was performed using the SPSS version 16.0 software (SPSS Inc., Chicago, IL, USA). The load-displacement data received from the device were used to calculate stiffness values by maximum displacement method (maximum load/maximum displacement; N/mm) (Figure 4). Statistical analyses were performed by Kruskal Wallis test to determine if a difference existed and Bonferroni-corrected Mann-Whitney $U$ test for isolated differences. No statistical tests were performed for assessing the normality of data distribution because of the nature

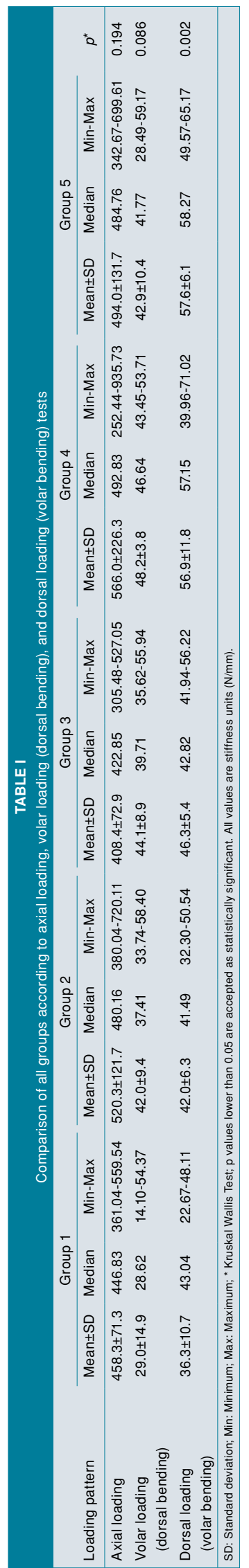


of the study (in vitro controlled study with small sample size). As Bonferroni-corrected Mann-Whitney $\mathrm{U}$ tests were used, the value of $p$ was accepted as alpha $=0.01$.

\section{RESULTS}

All samples in each of the five test groups were tested without catastrophic failure. Loaddisplacement curves in axial loading tests revealed minimal deviations due to closing of the $1 \mathrm{~mm}$ volar fracture gap. In dorsal and volar loading tests, the load-displacement curves were completely linear (Figure 4). None of the curves showed any characteristics of plastic deformation. Table I summarizes the mean structural stiffness levels of the groups.

Stiffness values from axial loading tests between groups in paired comparison showed no statistically significant difference (Table I, $\mathrm{p}=0.194$ ). Volar loading (dorsal bending) tests showed no statistically significant difference between the groups in pairwise comparison (Table I, $\mathrm{p}=0.086$, Kruskal Wallis test). Dorsal loading (volar bending) tests showed statistically significant difference between the groups in pairwise comparison (Table I, $\mathrm{p}=0.002$, Kruskal Wallis test). Three-pin groups (Groups 3, 4, and 5) had higher stiffness values compared to two-pin groups (Groups 1 and 2) in dorsal loading tests $(\mathrm{p}=0.000$, Mann-Whitney $U$ test with Bonferroni correction). Between groups comparisons demonstrated that Group 4 had higher values compared to Group 1 in dorsal loading tests ( $p=0.01$, Mann-Whitney $U$ test with Bonferroni correction). Group 5 performed better compared to Groups 1, 2, and 3 in dorsal loading tests $(p=0.01$, $p=0.002$, and $p=0.01$, respectively; Mann-Whitney $\mathrm{U}$ test with Bonferroni correction). Comparisons between Groups 4 and 5 and comparisons of Group 1 with Groups 2 and 3 showed no statistically significant difference $(\mathrm{p}>0.01)$.

\section{DISCUSSION}

In this study, stability of five different extra-focal bi-cortical pin configurations often used in our institution was evaluated. Three of five groups had three-pin configurations and two groups had two-pin configurations. Group 1 configuration was similar to Willenegger's method while other pin configurations were modified configurations which were not identical to methods which were previously described in the literature. In our study, no statistically significant difference was found between three-pin configurations (Kruskal Wallis, $\mathrm{p}>0.05$ and Bonferroni corrected Mann-Whitney $\mathrm{U}, \mathrm{p}>0.01)$. Likewise, there was no statistically significant difference between two-pin configurations ( $p>0.05)$. Additionally, there was no statistically significant difference in axial loading and volar loading tests $(p>0.01)$. However, there was a statistically significant difference between two- and three-pin configurations in favor of the latter group in dorsal loading tests $(p=0.000)$ in our study ${ }^{[17]}$ Group 4 configuration showed higher values than only Group 1 in dorsal loading tests $(\mathrm{p}=0.01)$. Nonetheless, Group 5 configuration was also found to have significantly higher stiffness values only in dorsal loading tests compared to two-pin configurations and Group $3(\mathrm{p}<0.01)$. Although not statistically significant, axial and volar loading stiffness values of Group 4 were higher than the two-pin configurations (Table I, $p>0.05$ ). However, clinically, dorsally comminuted extra-articular distal radius fractures mostly displace dorsally, and volar angulation is the least likely mode of failure in these patients..$^{[7,8]}$

Concerning the fixation of distal radius fractures with percutaneous pinning methods, most of the available studies are clinical studies comparing either (i) percutaneous pinning with plaster immobilization; ${ }^{[9]}$ (ii) the Kapandji method with trans-styloid fixation method;:[4] (iii) Kapandji method with Py method; ${ }^{[5,6]}$ (iv) modified Kapandji method with Willenegger method; ${ }^{[3]}$ and (v) complications of percutaneous pinning methods. ${ }^{[7,8]}$ Most of these studies indicated that percutaneous pinning methods cannot be used for all unstable distal radius fractures, and compared with plaster immobilization, percutaneous pinning is useful in preventing deformity and malunion, and that extra-focal pinning methods such as Willenegger and Py provide better results than intra-focal methods such as Kapandji. ${ }^{[8]}$ However, there are also studies reporting successful results with intra-focal methods. ${ }^{[3]}$ Barton et al. ${ }^{[18]}$ in a study on 53 patients after fixation with K-wires, found that this method perfectly controlled dorsal angulation; however, it was not very successful for preventing radial shortening. In a biomechanical study, Fritz et al. ${ }^{[19]}$ examined the Willenegger method, the Kapandji method, and a combination of these methods for dorsally unstable distal radius fractures. The authors found that in direct dorsal load tests, the Kapandji method provided more stable fixation than the Willenegger method, while Willenegger-type fixation caused a definite increase in stability in all directions and that the stability of the combined method was equal to the sum of the stability of each component. 
When reviewing the studies on biomechanical evaluation of the methods used in the surgical treatment of distal radius fractures, there is no clear consensus regarding loading protocols. Some of these studies used load-to-failure protocols while others preferred cyclic-loading or non-load-to-failure protocols like ours. In the early postoperative period, when the patient's arm is in a cast or splint, axial loading pattern due to compressive forces occurs with the flexion of the digits. During an early postoperative rehabilitation period, the compressive forces generated by combined low-strength active motion exercises of the wrist and digits do not exceed $250 \mathrm{~N}$ of axial loading, while these limited motions generate forces of axial compression, dorsal, and volar bending. ${ }^{[16,20]}$ Willis et al. ${ }^{[16]}$ compared the relative stability of five distal radius plates with the use of a sawbone fracture model under axial, dorsal, and volar loading conditions using a non-load-tofailure loading protocol. In our study, we performed preliminary tests using the same loading protocol and obtained linear load-displacement curves suggesting the elastic behavior of the entire fixed construct. In the preliminary phase of our study, we also used a video extensiometer to measure the relative fracture gap motion. However, since the data we obtained from this device were not consistent and not considered meaningful, we excluded these data from tests and used the data from the universal test device which shows the stiffness of the entire construct. As the preliminary tests showed linear load-displacement curves, we took for reference the loading protocol of Willis et al., ${ }^{[16]}$ as $250 \mathrm{~N}$ axial loading, and $50 \mathrm{~N}$ dorsal and volar loadings. As can be seen in the load-displacement curves, dorsal and volar loading measurements are completely linear, and rare small deviations in axial loading measurements are due to the closing of the $1 \mathrm{~mm}$ volar gap under axial compression. Another issue that may contribute to these deviations may be alterations of the force vector due to the closing of the wedge under axial compression. Eventually, our load-displacement curves do not resemble the characteristics of plastic deformation. In our study, we standardized the loading points in each specimen precisely; however, as the axis of rotation for each construct cannot be calculated and the loading points were not centered along these axes, we may admit that some degree of rotational and maybe translational forces may have occurred while testing.

In a study comparable to ours, Naidu et al. ${ }^{[21]}$ examined the effect of pin configurations and thickness of Kirschner wires. They applied torsional loading and dorsal bending and concluded that to ensure a stable fixation, at least $1.6 \mathrm{~mm}$ (0.062 inch) K-wires should be used and the most stable pin configuration had two radial styloid pins with an additional pin from the ulnar corner (identical to Group 3 configuration in our study). Their study did not include groups having the convergent or divergent pins from the styloid like the Groups 4 and 5 in our study. Our goal was to investigate the resistance against radial shortening under axial compression when including these non-parallel pin configurations. In our study, Group 5 configuration showed significant difference compared to two-pin configurations (Groups 1 and 2) and Group 3 under dorsal loading $(\mathrm{p}<0.01)$.

Because of their inherent properties, fourthgeneration composite bone models have been widely used in mechanical testing of fracture fixation constructs. These composite models have high consistency in most mechanical and anatomical properties facilitating standardized tests with minimal inter-specimen variability. ${ }^{[22,23]}$ However, some authors prefer a two-phase test setup with synthetic and cadaveric bone samples. ${ }^{[23]}$

In a meta-analysis examining dorsally displaced distal radius fractures treated with percutaneous pinning, Handoll et al. ${ }^{[8]}$ concluded that low-level evidence existed to support percutaneous pinning and the role and method are not supported by validated studies. In addition, evidence to determine whether to use two- or three-pin method is insufficient as no studies were qualified to address this question. ${ }^{[13,24]}$ Likewise, by means of statistical significance, our data showed no pronounced and clinically significant difference between the test groups.

Nevertheless, in selected cases of unstable distal radius fracture with dorsal comminution, closed reduction and extra-focal percutaneous pinning may be an option as a less invasive and low-cost method for surgical treatment. In the present study, we found that configurations of Groups 4 and 5 demonstrated better stiffness values compared to two-pin configurations in dorsal loading tests.

Although this study is a controlled in vitro study performed using sawbones with minimal anatomic and mechanical variances, some missing elements should be addressed. First, the experimental setup did not include the soft tissue components. Finite element models or cadaver studies including these components may be suggested for this purpose. Second, we only analyzed axial, dorsal, and volar loading conditions, rotational and translational forces 
may occur under physiologic conditions. However, as mentioned before, some degree of rotational and translational loading may have occurred during tests. And third, although fourth generation sawbones are manufactured specifically for such mechanical tests, one type of sawbones cannot simulate all situations (i.e., osteoporosis).

In conclusion, if percutaneous pinning is the treatment of choice, we suggest the use of a three-pin construct. Particularly in cases with a risk of volar angulation, we recommend a three-pin configuration with two convergent or divergent bi-cortical K-wires as described in Group 4 or preferably Group 5.

\section{Declaration of conflicting interests}

The authors declared no conflicts of interest with respect to the authorship and/or publication of this article.

\section{Funding}

The authors received no financial support for the research and/or authorship of this article.

\section{REFERENCES}

1. Talmaç MA, Görgel MA, Kanar M, Tok O, Özdemir HM. Comparison of three surgical methods in the treatment of intraarticular comminuted distal radius fractures: Volar locking plate, non-bridging external fixator, and bridging external fixator. Eklem Hastalik Cerrahisi 2019;30:224-32.

2. Miller JD, Riley SA. Current concepts in pediatric distal radial fractures. Curr Orthop Pract 2012;23:409-13.

3. Strohm PC, Müller CA, Boll T, Pfister U. Two procedures for Kirschner wire osteosynthesis of distal radial fractures. A randomized trial. J Bone Joint Surg [Am] 2004;86:2621-8.

4. Lenoble E, Dumontier C, Goutallier D, Apoil A. Fracture of the distal radius. A prospective comparison between trans-styloid and Kapandji fixations. J Bone Joint Surg [Br] 1995;77:562-7.

5. Fikry T, Fadili M, Harfaoui A, Dkhissi M, Zryouil B. Metaphysis fracture of the distal radius: Kapandji's or Py's pinning?. Ann Chir Main Memb Super 1998;17:31-40.

6. Saddiki R, Ohl X, Hemery X, Vitry F, Dehoux E, Harisboure A. Dorsally displaced distal radius fractures: comparative study of Py's and Kapandji's techniques. Orthop Traumatol Surg Res 2012;98:61-7.

7. Diaz-Garcia RJ, Oda T, Shauver MJ, Chung KC. A systematic review of outcomes and complications of treating unstable distal radius fractures in the elderly. J Hand Surg Am 2011;36:824-35.e2.

8. Handoll HH, Vaghela MV, Madhok R. Percutaneous pinning for treating distal radial fractures in adults. Cochrane Database Syst Rev 2007;(3):CD006080.

9. Azzopardi T, Ehrendorfer S, Coulton T, Abela M. Unstable extra-articular fractures of the distal radius: a prospective, randomised study of immobilisation in a cast versus supplementary percutaneous pinning. J Bone Joint Surg [Br] 2005;87:837-40.

10. Saeki $Y$, Hashizume $H$, Nagoshi $M$, Tanaka $H$, Inoue H. Mechanical strength of intramedullary pinning and transfragmental Kirschner wire fixation for Colles' fractures. J Hand Surg Br 2001;26:550-5.

11. Rupp M, Cambon-Binder A, Alt V, Feron JM. Is percutaneous pinning an outdated technique for distal radius fractures? Injury 2019;50 Suppl 1:S30-5.

12. Chaudhry H, Kleinlugtenbelt YV, Mundi R, Ristevski B, Goslings JC, Bhandari M. Are volar locking plates superior to percutaneous k-wires for distal radius fractures? A metaanalysis. Clin Orthop Relat Res 2015;473:3017-27.

13. The treatment of distal radius fractures: guideline and evidence report. Adopted by the American Academy of Orthopaedic Surgeons board of directors. 2009. Available at: http://www.aaos.org/research/guidelines/drfguideline. pdf. [Accessed: June, 2020].

14. Doumas C, Bozentka DJ. K-wire fixation of distal radius fractures with and without external fixation. In: Hunt TR, Flynn JM, Wiesel SW, editors. Operative techniques in hand, wrist, and forearm surgery. Philadelphia, PA: Lippincott Williams \& Wilkins; 2011. p. 70-9.

15. Husby T. Closed reduction and percutanous pinning. In: Hove LM, Lindau T, Hølmer P, editors. Distal radius fractures current concepts. Berlin. Springer Verlag; 2014. p. 139-45.

16. Willis AA, Kutsumi K, Zobitz ME, Cooney WP 3rd. Internal fixation of dorsally displaced fractures of the distal part of the radius. A biomechanical analysis of volar plate fracture stability. J Bone Joint Surg [Am] 2006;88:2411-7.

17. Atik OŞ. What are the expectations of an editor from a scientific article? Jt Dis Relat Surg 2020;31:597-8.

18. Barton T, Chambers C, Lane E, Bannister G. Do Kirschner wires maintain reduction of displaced Colles' fractures? Injury 2005;36:1431-4.

19. Fritz T, Heyer T, Krieglstein C, Mattern R, Kallieris D, Friedl W. Biomechanik der kombinierten KirschnerdrahtOsteosynthese am humanen Modell der dorsal instabilen, distalen Radiusfraktur (Colles-Typ) [Biomechanics of combined Kirschner wire osteosynthesis in the human model of unstable dorsal, distal radius fractures (Colles type)]. Chirurg 1997;68:496-502.

20. Graham TJ, Louis DS. Biomechanical aspects of percutaneous pinning for distal radial fractures. In: Saffar P, Cooney WP, editors. Fractures of the distal radius. London: Martin-Dunitz Ltd; 1995. p. 28-36.

21. Naidu SH, Capo JT, Moulton M, Ciccone W 2nd, Radin A. Percutaneous pinning of distal radius fractures: a biomechanical study. J Hand Surg Am 1997;22:252-7.

22. Elfar J, Menorca RM, Reed JD, Stanbury S. Composite bone models in orthopaedic surgery research and education. J Am Acad Orthop Surg 2014;22:111-20.

23. Hausmann, J. Sawbones in Biomechanical Settings a Review. Osteosynthesis and Trauma Care 2006;14:259-64.

24. Koval K, Haidukewych GJ, Service B, Zirgibel BJ. Controversies in the management of distal radius fractures. J Am Acad Orthop Surg 2014;22:566-75. 